\title{
The Effect of Tobacco Smoking on Periodontal Health ${ }^{\dagger}$
}

\author{
Dorina Lauritano 1,*, Giulia Moreo ${ }^{1}$, Francesco Carinci ${ }^{2}$, Alberta Lucchese ${ }^{3}$, Dario di Stasio ${ }^{3}$, \\ Fedora della Vella ${ }^{4}$ and Massimo Petruzzi ${ }^{4}$ \\ 1 Department of Medicine and Surgery, Centre of Neuroscience, University of Milano-Bicocca, \\ 20126 Milan, Italy; moreo.giulia@gmail.com \\ 2 Department of Morphology, Surgery and Experimental Medicine, University of Ferrara, \\ 44121 Ferrara, Italy; crc@unife.it \\ 3 Multidisciplinary Department of Medical-Surgical and Dental Specialties, University of Campania-Luigi \\ Vanvitelli, 80138 Naples, Italy; alberta.lucchese@unicampania.it (A.L.); \\ dario.distasio@unicampania.it (D.d.S.) \\ 4 Interdisciplinary Department of Medicine, University of Bari, 70121 Bari, Italy; \\ fdellavella@gmail.com (F.d.V.); massimo.petruzzi@uniba.it (M.P.) \\ * Correspondence: dorina.lauritano@unimib.it; Tel.: +39-335-679-0163 \\ + Presented at the XV National and III International Congress of the Italian Society of Oral Pathology and \\ Medicine (SIPMO), Bari, Italy, 17-19 October 2019.
}

Published: 10 December 2019

Periodontal diseases (PD) affect about half of the adult population all over the world. PD is caused by bacterial infection inducing an inflammatory response with progressive destruction of the periodontal tissues and finally the lost of teeth. Tobacco smoking (TS), alcohol consumption, and systemic conditions such as diabetes, osteoporosis, malnutrition and stress are considered additional risk factors [1,2]. This short review examines the potential causal association between TS and PD. There are many studies for a higher level of PD among smokers. Greater levels of clinical alveolar bone loss, tooth mobility, probing pocket depth and tooth loss are more frequent in smokers than in non-smokers. The modification of the periodontitis micro-flora in smokers influences the development of PD. Also, there are data suggesting smoking effects on both host responses in humans. Response to periodontal treatment is different in smokers and non-smokers. Various clinical studies have demonstrated that TS is a major risk factor for poor response to periodontal therapy. TS is a factor that has the potential to negatively affect healing and the outcome of implant treatment [3]. It is mandatory for dentists and dental hygienist to promote smoking-cessation programs as well as educate our community on the benefits of not smoking $[3,4]$.

Conflicts of Interest: The authors declare no conflict of interest.

\section{References}

1. American Academy of Periodontology. International workshop for classification of periodontal diseases and conditions. Ann. Periodontol. 1999, 4, 7-112.

2. Blum, A.; Front, E.; Peleg, A. Periodontal care may improve systemic inflammation. Clin. Investig. Med. 2007, 30, E114-E117.

3. Samet, J.M.; Wipfli, H.L. Globe still in grip of addiction. Nature 2010, 463, 1020-1021.

4. Jha, P.; Chaloupka, F.J.; Corrao, M.; Jacob, B. Reducing the burden of smoking world-wide: Effectiveness of interventions and their coverage. Drug Alcohol Rev. 2006, 25, 597-609. 